\title{
Yet more versions of $d=11$ supergravity
}

\author{
B Drabant $\dagger, M$ Töx $\dagger$ and $\mathrm{H}$ Nicolai $\dagger$ \\ $\dagger$ Institut für Theoretische Physik, Universität Karlsruhe, D-7500 Karlsruhe 1, Federal \\ Republic of Germany \\ $\ddagger$ Institut für Theoretische Physik, Universität Hamburg, D-2000 Hamburg 50, Federal \\ Republic of Germany
}

Received 26 September 1988

\begin{abstract}
Extending previous results, we construct two further versions of $d=11$ supergravity. The transformation laws are given in a form where the $\operatorname{SO}(1,10)$ local Lorentz group is replaced by $S O(1,4) \times U S p(8)$ and $S O(1,5) \times[S O(5) \times S O(5)]$, respectively. The spinless fields are assigned to the corresponding representations of $E_{6(+6)}$ and $E_{5(+5)}$.
\end{abstract}

\section{Introduction}

It has been known for some time that maximally extended supergravities in $D$ dimensions obtained from $d=11$ supergravity [1] are manifestly invariant under an internal symmetry group $\mathrm{SL}(11-D)_{\text {global }} \times \mathrm{SO}(11-D)_{\text {local }}[2,3]$. This symmetry can be enlarged to the product of a non-compact global group $\mathrm{G}_{11-D}$ and a compact local group $\mathrm{H}_{11-D}$. Assuming that the scalar sector in these theories is governed by a non-linear $\sigma$ model based on the coset $\mathrm{G}_{11-D} / \mathrm{H}_{11-D}$, one can deduce the relevant groups by equating the number of scalars obtained in the dimensional reduction with the dimension of this coset space. In this way, one gets $[2,3]$ the results presented in table $1 \dagger$.

In [4] it was shown that some of these hidden symmetries are actually present in the $d=11$ supergravity itself: there exists a reformulation where the tangent space group $\mathrm{SO}(1,3) \times \mathrm{SO}(7)$ is replaced by $\mathrm{SO}(1,3) \times \mathrm{SU}(8)$ and the spinless degrees of freedom are assigned to a representation of $E_{7(+7)}$. A crucial step in this construction

Table 1.

\begin{tabular}{lll}
\hline$D$ & $\mathrm{G}_{11-D}$ & $\mathrm{H}_{11-D}$ \\
\hline 8 & $\mathrm{E}_{3(+3)}=\mathrm{SL}(3, \mathbb{R}) \times \mathrm{SL}(2, \mathbb{R})$ & $\mathrm{SO}(3) \times \mathrm{SO}(2)$ \\
7 & $\mathrm{E}_{4(+4)}=\mathrm{SL}(5, \mathbb{R})$ & $\mathrm{SO}(5)$ \\
6 & $\mathrm{E}_{5(+5)}=\mathrm{SO}(5,5)$ & $\mathrm{SO}(5) \times \operatorname{SO}(5)$ \\
5 & $\mathrm{E}_{6(+6)}$ & $\mathrm{USp}(8)$ \\
4 & $\mathrm{E}_{7(+7)}$ & $\mathrm{SU}(8)$ \\
3 & $\mathrm{E}_{8(+8)}$ & $\mathrm{SO}(16)$ \\
\hline
\end{tabular}

$\dagger$ For $D \geqslant 9$ the field $A_{M N P}$ does not give any scalars in the reduction. Hence one retains only $G L(2, \mathbb{R})_{g l o b a l} \times$ $\mathrm{SO}(2)_{\text {local }}$ after the Weyl rescaling for $D=9$. 
was the introduction of 'composite' gauge connections in the internal dimensions so as to covariantise the theory with respect to $S U(8)$ transformations depending on the internal coordinates. Subsequently, the corresponding version of $d=11$ supergravity with tangent space group $\mathrm{SO}(1,2) \times \mathrm{SO}(16)$ was constructed in [5]. These results suggest that, in fact, all of the hidden symmetries displayed in table 1 can be realised in the full $d=11$ theory. In this paper we show that this is indeed the case by presenting explicit results for $D=5$ and $D=6$ (we have not considered $D \geqslant 7$ since these cases are less interesting and unlikely to furnish any new insights). This confirms the conclusion that $d=11$ supergravity is a multifaceted theory whose ultimate formulation (and possible embedding into an ancestor dual model) remains to be found. It would, of course, be even more interesting if one could extend the results now established for $3 \leqslant D \leqslant 6$ to $D \leqslant 2$ where one expects the emergence of infinite-dimensional groups $\uparrow$.

We now briefly summarise our notation and conventions; more details can be found in $[4,5]$. The only difference with $[4,5]$ is that we use a metric with signature $(+,-, \ldots,-)$. The Lagrangian of $d=11$ supergravity is given by $\eta$

$$
\begin{array}{rl}
\mathscr{L}=-\frac{1}{2} E R-\frac{1}{2} \mathrm{i} & E \bar{\Psi}_{M} \tilde{\Gamma}^{M N P} D_{N} \Psi_{P}-\frac{1}{48} E F_{M N P Q}^{2} \\
& +(1 / \sqrt{2})\left[1 /(12)^{3}\right] \varepsilon^{M \ldots X} F_{M N P Q} F_{R S T U} A_{V W X} \\
& +(\sqrt{2} / 192) E F_{M N P Q}\left(\bar{\Psi}_{R} \tilde{\Gamma}^{M N P Q R S} \Psi_{S}+12 \bar{\Psi}^{M} \tilde{\Gamma}^{N P} \Psi^{Q}\right)
\end{array}
$$

where, of course,

$$
\left\{\tilde{\Gamma}^{A}, \tilde{\Gamma}^{B}\right\}=2 \eta^{A B} \quad \tilde{\Gamma}^{A_{1} \ldots A_{11}}=-\mathrm{i} \varepsilon^{A_{1} \ldots A_{11}} .
$$

The transformation laws are

$$
\begin{aligned}
& \delta E_{M}^{A}=-\frac{1}{2} \mathrm{i} \bar{\varepsilon} \tilde{\Gamma}^{A} \Psi_{M} \\
& \delta A_{M N P}=\frac{1}{8} \sqrt{2} \bar{\varepsilon} \tilde{\Gamma}_{[M N} \Psi_{P]} \\
& \delta \Psi_{M}=D_{M} \varepsilon+\frac{1}{288} \mathrm{i} \sqrt{2}\left(\tilde{\Gamma}_{M}{ }^{P Q R S}-8 \delta_{M}^{P} \tilde{\Gamma}^{Q R S}\right) \varepsilon F_{P Q R S} .
\end{aligned}
$$

Higher-order fermionic terms are neglected throughout.

\section{Field redefinitions and transformation laws}

The starting point is a $D+(11-D)$ coordinate split. The curved and flat $d=11$ indices $M$ and $A$ are decomposed into $(\mu, m)$ and $(\alpha, a)$, respectively. Fixing the gauge

$$
E_{M}{ }^{A}=\left(\begin{array}{cc}
e_{\mu}^{\alpha} & B_{\mu}{ }^{m} e_{m}{ }^{a} \\
0 & e_{m}{ }^{a}
\end{array}\right)
$$

reduces the local $\mathrm{SO}(1,10)$ Lorentz group to $\mathrm{SO}(1, D-1) \times \mathrm{SO}(11-D)$. The Weyl rescaling

$$
e_{\mu}^{\prime \alpha}=\Delta^{s} e_{\mu}^{\alpha} \quad \Delta \equiv \operatorname{det} e_{m}^{a} \quad s \equiv 1 /(D-2)
$$

leads to the standard Einstein action in $D$ dimensions (for $D>2$ ). The fermionic fields are redefined as follows:

$$
\begin{aligned}
& \Psi_{\mu}^{\prime}=\Delta^{-s / 2} e_{\mu}^{\prime \alpha}\left(\Psi_{\alpha}+s \tilde{\Gamma}_{\alpha} \tilde{\Gamma}^{a} \Psi_{a}\right)=e_{\mu}^{\prime \alpha} \Psi_{\alpha}^{\prime} \\
& \Psi_{a}^{\prime}=\Delta^{-s / 2} \Psi_{a} \\
& \varepsilon^{\prime}=\Delta^{s / 2} \varepsilon .
\end{aligned}
$$

\footnotetext{
† For some recent results concerning maximally extended supergravity in two dimensions, see [6].
} 
As a general rule, quantities with index $\mu$ are obtained from the flat ones via $e_{\mu}{ }^{\alpha}$ and without the $E_{\mu}{ }^{a}$ contribution (so that we have a scalar under $(11-D)$-dimensional general coordinate transformations $\left.\xi^{m}\left(x^{\mu}, y^{m}\right)\right)$. In particular, we use $[4,5]$

$$
\begin{aligned}
& \mathscr{D}_{\mu}=e_{\mu}{ }^{\alpha} \partial_{\alpha}=\partial_{\mu}-B_{\mu}{ }^{m} \partial_{m} \\
& \omega_{\mu A B}=\frac{1}{2} e_{\mu}{ }^{\alpha}\left(\Omega_{A B \alpha}-\Omega_{B \alpha A}-\Omega_{\alpha A B}\right)
\end{aligned}
$$

where

$$
\Omega_{A B}{ }^{C}=2 E_{[A}^{M} E_{B]}{ }^{N} \partial_{M} E_{N}{ }^{C} .
$$

The supersymmetry transformation of the spin-2 field is given by

$$
\begin{aligned}
\delta e_{\mu}^{\prime \alpha} & =\delta_{\text {susy }}\left(\Delta^{s} e_{\mu}{ }^{\alpha}\right)+E_{\mu}{ }^{A} \Omega_{A}{ }^{\alpha} \\
& =-\frac{1}{2} \mathbf{i} \bar{\varepsilon}^{\prime} \tilde{\Gamma}^{\alpha} \Psi_{\mu}^{\prime} .
\end{aligned}
$$

The compensating $\mathrm{SO}(1,10)$ rotation

$$
\Omega_{a \alpha}=\frac{1}{2} \mathrm{i} \bar{\varepsilon} \tilde{\Gamma}_{\alpha} \Psi_{a} \quad \Omega_{\alpha \beta}=\frac{1}{2} \mathrm{i} s \bar{\varepsilon} \tilde{\Gamma}_{\alpha \beta} \tilde{\Gamma}^{a} \Psi_{a}
$$

restores the gauge (2.1). The spin-1 fields transform into

$$
\delta B_{\mu}{ }^{m}=-\frac{1}{2} \mathrm{i} \Delta^{-s} e_{a}^{m}\left[\bar{\varepsilon}^{\prime} \tilde{\Gamma}^{a} \Psi_{\mu}^{\prime}+\bar{\varepsilon}^{\prime} \tilde{\Gamma}^{\prime \mu}\left(\eta^{a b}+s \tilde{\Gamma}^{a} \tilde{\Gamma}^{b}\right) \Psi_{b}^{\prime}\right]
$$

Variation of the spin $-\frac{3}{2}$ fields $\Psi_{\mu}^{\prime}(2.3)$ yields the lengthy expression

$$
\begin{aligned}
\delta \Psi_{\mu}^{\prime}=\left[\mathscr{D}_{\mu}-\right. & \left.\frac{1}{4}\left(\hat{\omega}_{\mu \alpha \beta} \tilde{\Gamma}^{\alpha \beta}+\omega_{\mu a b}^{\prime} \tilde{\Gamma}^{a b}\right)-\frac{1}{2} s \partial_{m} B_{\mu}^{m}\right] \varepsilon^{\prime} \\
& +\left(\frac{1}{2} s \tilde{\Gamma}_{\mu}^{\prime} \tilde{\Gamma}^{\alpha \beta}-e_{\mu}^{\prime[\alpha} \tilde{\Gamma}^{\beta]}\right)\left(-\frac{1}{4} \Delta^{s} \Omega_{\alpha \beta a}^{\prime} \tilde{\Gamma}^{a}+\frac{1}{8} \sqrt{2} \Delta^{-s} F_{\alpha \beta c d} \tilde{\Gamma}^{c d}\right) \varepsilon^{\prime} \\
& -s \Delta^{-s} e_{a}^{m} \tilde{\Gamma}^{a}\left[\partial_{m}+\frac{1}{4} \Omega_{m \alpha \beta}^{\prime} \tilde{\Gamma}^{\alpha \beta}-\frac{1}{4} \omega_{m a b} \tilde{\Gamma}^{a b}-\frac{1}{2}(1+s)\left(\Delta^{-1} \partial_{m} \Delta\right)\right] \tilde{\Gamma}_{\mu}^{\prime} \varepsilon^{\prime} \\
& +\left(\frac{1}{2}-s\right) \Omega_{a(\alpha \beta)}^{\prime} e_{\mu}^{\prime \alpha} \tilde{\Gamma}^{\beta} \tilde{\Gamma}^{a} \varepsilon^{\prime}+\frac{1}{96} \sqrt{2} \Delta^{-s} e_{\mu}^{\prime \alpha}\left[-s F_{a b c d} \tilde{\Gamma}_{\alpha} \tilde{\Gamma}^{a b c d}-4 F_{\alpha b c d} \tilde{\Gamma}^{b c d}\right. \\
& \left.+\left(8 s \tilde{\Gamma}_{\alpha} \tilde{\Gamma}^{\beta \gamma \delta}-12 \delta_{\alpha}^{[\beta} \tilde{\Gamma}^{\gamma \delta]}\right) F_{\beta \gamma \delta d} \tilde{\Gamma}^{d}+\left(3 s \tilde{\Gamma}_{\alpha} \tilde{\Gamma}^{\beta \gamma \delta \varepsilon}-4 \delta_{\alpha}^{[\beta} \tilde{\Gamma}^{\gamma \delta \varepsilon]}\right) F_{\beta \gamma \delta \varepsilon}\right] \varepsilon^{\prime} \\
& \hat{\omega}_{\mu \alpha \beta} \equiv \omega_{\mu \alpha \beta}^{\prime}+2 s e_{\mu[\alpha}^{\prime} e_{\beta]^{\prime} d_{m}}^{\prime \prime} B_{\nu}^{m} .
\end{aligned}
$$

\section{Covariantisation for $D=5$}

To establish local USp $(8)$ covariance we give some notation and definitions for the $5+6$ coordinate split. The $d=11 \gamma$ matrices are represented by

$$
\begin{array}{ll}
\tilde{\Gamma}^{\alpha}=\gamma^{\alpha} \otimes \Gamma^{*} & \Gamma^{*}=\mathrm{i} \Gamma^{123456}=\mathrm{i} \Omega=\mathrm{i} \Gamma^{7} \\
\tilde{\Gamma}^{a}=1 \otimes \Gamma^{a} . &
\end{array}
$$

For $\gamma^{\alpha}(\alpha=0, \ldots, 4)$ we use the conventions of [3]. $\Gamma^{a}$ and $\Omega$ are real antisymmetric $8 \times 8$ matrices:

$$
\left\{\Gamma^{a}, \Gamma^{b}\right\}=2 \eta^{a b} 1 \quad \Omega^{2}=-1 .
$$

Accordingly, the $D=11$ Dirac indices are split as $(\underline{\alpha}, A)$ where $\underline{\alpha}$ is the $D=5$ index and $A$ is the 'internal' $\mathrm{SO}(6)$ spinor index. We will suppress the index $\underline{\alpha}$ in the following. The $\mathrm{SO}(6)$ part of the local Lorentz group acting on Majorana spinors can be extended to USp $(8)$ with the 36 generators $\Omega, \Gamma^{a b}$, i $\Gamma^{a b c}$ satisfying

$$
\begin{aligned}
& \Lambda^{+}=-\Lambda \\
& (\Omega \Lambda)^{\top}=\Omega \Lambda .
\end{aligned}
$$


We can raise and lower $\operatorname{USp}(8)$ indices by means of the symplectic metric $\Omega$. Thus

$$
\Psi_{A}=\Omega_{A B} \Psi^{B}
$$

transforms in the complex conjugate representation of $\Psi^{B}$ by virtue of (3.3). It follows that $\mathrm{USp}(8)$ is compatible with the $d=11$ Majorana condition which in turn is equivalent to the $D=5$ pseudo-Majorana condition [3]

$$
\Psi^{A}=\gamma_{5} \Omega_{A B}\left(\Psi^{B}\right)^{*} .
$$

The next step is to regroup the 48 spin- $\frac{1}{2}$ fields $\Psi_{a}{ }^{A}$ into a representation of USp(8). This is done as follows $[2,3]$ :

$$
\begin{aligned}
\chi_{A B C} & \equiv \frac{3}{2} \sqrt{2} i \Gamma_{\langle A B}^{a} \Psi_{C \backslash a}^{\prime} \\
& =\frac{3}{2} \sqrt{2} i\left\{\Gamma_{[A B}^{a} \Psi_{C] a}^{\prime}+\frac{1}{3} \Omega_{[A B} \Gamma_{C] D}^{a} \Psi_{a}^{\prime D}\right\}
\end{aligned}
$$

where $\langle\ldots\rangle$ denotes antisymmetric tensors which are traceless with respect to $\Omega . \chi_{A B C}$ satisfies the reality condition

$$
\chi^{A B C}=\gamma_{5}\left(\chi_{A B C}\right)^{*} \text {. }
$$

We note the identity

$$
\bar{\chi}^{A B C} \gamma^{\mu} D_{\mu} \chi_{A B C}=6 \mathrm{i} \bar{\Psi}_{a}\left(\eta^{a b}+\frac{1}{3} \Gamma^{a} \Gamma^{b}\right) \tilde{\Gamma}^{\mu} D_{\mu} \Psi_{b}
$$

which renders the spin- $\frac{1}{2}$ kinetic term diagonal.

From (2.5) and (2.7) we obtain the USp(8) covariant transformation laws

$$
\begin{aligned}
& \delta e_{\mu}^{\prime \alpha}=\frac{1}{2} \bar{\varepsilon}^{A} \gamma^{\alpha} \Psi_{\mu A} \\
& \delta B_{\mu}^{m}=-\frac{1}{2} e_{A B}^{m}\left(\bar{\varepsilon}^{A} \Psi_{\mu}^{B}-\frac{1}{4} \sqrt{2} \bar{\varepsilon}_{C} \gamma_{\mu}^{\prime} \chi^{A B C}\right)
\end{aligned}
$$

where the generalised vielbein $e_{A B}^{m}$ is defined as

$$
e_{A B}^{m} \equiv \mathrm{i} \Delta^{-1 / 3} e_{a}^{m} \Gamma_{A B}^{a}
$$

and thereby assigned to the 27 representation of $U S p(8)$. As explained in [4] one must introduce a local USp(8) to avoid the emergence of new degrees of freedom; $(3.10)$ then corresponds to a special gauge choice. Raising indices gives $e^{m A B}=\left(e_{A B}^{m}\right)^{*}$.

Using properties of the $d=7$ Clifford algebra $[2,4]$ the supersymmetry transformation of $e_{A B}^{m}$ can be cast into the form

$$
\delta_{\text {susy }} e_{A B}^{m}=\frac{1}{2} \sqrt{2} \Sigma_{A B C D} e^{m C D}+2 \Lambda_{[A}^{C} e_{B] C}^{m}
$$

with

$$
\Sigma_{A B C D}=\bar{\varepsilon}_{\left[A \chi_{B C D]}\right.}+\frac{1}{24} \varepsilon_{A B C D E F G H} \bar{\varepsilon}^{E} \chi^{F G H}
$$

and the USp(8) parameter

$$
\Lambda_{B}^{A}=-\frac{1}{8} \mathrm{i}\left(\bar{\varepsilon} \Gamma_{a} \Psi_{b}\right) \Gamma^{a b}+\frac{1}{16} \mathrm{i}\left(\bar{\varepsilon} \Gamma_{a b} \Psi_{c}\right) \Gamma^{a b c} .
$$

$\Sigma_{A B C D}$ is antisymmetric, traceless and pseudoreal: $\Sigma^{A B C D}=\left(\Sigma_{A B C D}\right)^{*}$ and thus transforms in the 42 representation of $\mathrm{USp}(8)$. Discarding the local $\mathrm{USp}(8)$ rotation we arrive at a manifestly covariant transformation law for $e_{A B}^{m}$. Furthermore the right-hand side of (3.11) can be interpreted as a (local) $E_{6}$ rotation in the fundamental 27 representation. 
We must then rewrite the fermionic variations. Just as in $[4,5],(2.8)$ can be simplified by the introduction of further $\operatorname{USp}(8)$ covariant quantities. These are found in exactly the same fashion as in $[4,5]$ and are given by (we omit all primes in the following)

$$
\begin{gathered}
\mathscr{B}_{\mu}{ }^{A}{ }_{B}=-\frac{1}{2} \omega_{\mu a b} \Gamma_{A B}^{a b}-\frac{1}{12} \sqrt{2} \mathrm{i} \Delta^{-1 / 3} e_{\mu}{ }^{\alpha} F_{\alpha b c d} \Gamma_{A B}^{b c d}-\frac{1}{48} \sqrt{2} \Delta^{-1 / 3} e_{\mu}{ }^{\alpha} \varepsilon_{\alpha \beta \gamma \delta \varepsilon} F^{\beta \gamma \delta \varepsilon} \Omega_{A B} \\
\mathscr{A}_{\mu A B C D}=\frac{3}{4} \Omega_{\mu(a b)} \Gamma_{(A B}^{a} \Gamma_{C D\rangle}^{b}-\frac{1}{8} \sqrt{2} \mathrm{i} \Delta^{-1 / 3} e_{\mu}{ }^{\alpha} F_{\alpha a b c} \Gamma_{[A B}^{a} \Gamma_{C D]}^{b c} \\
-\frac{1}{192} \sqrt{2} \Delta^{-1 / 3} e_{\mu}{ }^{\alpha} \varepsilon_{\alpha \beta \gamma \delta \varepsilon} F^{\beta \gamma \delta \varepsilon} \Gamma_{a[A B} \Gamma_{C D]}^{a 7} \\
\mathscr{B}_{m}{ }^{A}{ }_{B}=\frac{1}{2} e_{a}{ }^{n} \partial_{m} e_{n b} \Gamma_{A B}^{a b}-\frac{1}{48} \sqrt{2} i e_{m}{ }^{a} F_{a b c d} \Gamma_{A B}^{b c d} \\
\mathscr{A}_{m A B C D}=\frac{3}{4} e_{a}{ }^{n} \partial_{m} e_{n b} \Gamma_{\langle A B}^{a} \Gamma_{C D\rangle}^{b}-\frac{1}{32} \sqrt{2} i e_{m}{ }^{a} F_{a b c d} \Gamma_{[A B}^{b} \Gamma_{C D]}^{c d} .
\end{gathered}
$$

In addition we introduce the spin-1 field strength

$\mathscr{G}_{\alpha \beta}{ }^{A}{ }_{B}=\frac{1}{4} \mathrm{i} \Delta^{1 / 3} \Omega_{\alpha \beta a} \Gamma_{A B}^{a 7}-\frac{1}{8} \sqrt{2} \Delta^{-1 / 3} F_{\alpha \beta c d} \Gamma_{A B}^{c d 7}+\frac{1}{24} \sqrt{2} \mathbf{i} \Delta^{-1 / 3} \varepsilon_{\alpha \beta \gamma \delta \varepsilon} F_{a}^{\gamma \delta \varepsilon} \Gamma_{A B}^{a}$.

The USp $(8)$ covariant 'generalised vielbein postulate' is

$\mathscr{D}_{\mu} e_{A B}^{m}+\frac{1}{3} \partial_{n} B_{\mu}^{n} e_{A B}^{m}+\partial_{n} B_{\mu}^{m} e_{A B}^{n}+\mathscr{B}_{\mu}{ }^{C}{ }_{[A} e_{B] C}^{m}+\mathscr{A}_{\mu} A B C D e^{m C D}=0$

$\partial_{m} e_{A B}^{n}+\mathscr{B}_{m}{ }^{C}{ }_{[A} e_{B] C}^{n}+\mathscr{A}_{m A B C D} e^{n C D}=0$.

With the help of the $\mathrm{SO}(1,4) \times \mathrm{USp}(8)$ covariant derivatives

$$
\begin{aligned}
& D_{\mu} \varepsilon^{A} \equiv \mathscr{D}_{\mu} \varepsilon^{A}-\frac{1}{4} \hat{\omega}_{\mu \alpha \beta} \gamma^{\alpha \beta} \varepsilon^{A}+\frac{1}{2} \mathscr{B}_{\mu}{ }^{A}{ }_{B} \varepsilon^{B} \\
& D_{m} \varepsilon^{A} \equiv \partial_{m} \varepsilon^{A}+\frac{1}{4} e_{\alpha}^{\mu} \partial_{m} e_{\mu \beta} \gamma^{\alpha \beta} \varepsilon^{A}+\frac{1}{2} \mathscr{B}_{m}{ }^{A}{ }_{B} \varepsilon^{B}
\end{aligned}
$$

one finally obtains

$$
\begin{gathered}
\delta \chi_{A B C}=\frac{3}{2} \sqrt{2} e_{\langle A B}^{m} D_{m} \varepsilon_{C\rangle}-\frac{3}{4} \sqrt{2} \gamma^{\alpha \beta} \mathscr{G}_{\alpha \beta\langle A B} \varepsilon_{C\rangle}-\sqrt{2} \gamma^{\mu} \mathscr{A}_{\mu A B C D} \varepsilon^{D} \\
-\frac{3}{4} \sqrt{2} e^{m D E} \mathscr{A}_{m D E\langle A B} \varepsilon_{C\rangle}-\sqrt{2} \mathscr{A}_{m A B C D} e^{m D E} \varepsilon_{E} \\
\delta \Psi_{\mu}^{A}=\left[D_{\mu}\left(\hat{\omega}, \mathscr{B}_{\mu}\right)-\frac{1}{6} \partial_{m} B_{\mu}^{m}\right] \varepsilon^{A}+\frac{1}{6}\left(\gamma^{\alpha \beta} \gamma_{\mu}-2 e_{\mu}{ }^{\alpha} \gamma^{\beta}\right) \mathscr{G}_{\alpha \beta}{ }^{A}{ }_{B} \varepsilon^{B} \\
+\frac{1}{3} e^{m A B}\left[D_{m}\left(\gamma_{\mu} \varepsilon_{B}\right)+\frac{1}{4}\left(\partial_{m} g_{\mu \nu}\right) \gamma^{\nu} \varepsilon_{B}\right]-\frac{1}{6} e_{C D}^{m} \mathscr{A}_{m}^{A B C D} \gamma_{\mu} \varepsilon_{B} .
\end{gathered}
$$

In the reduction to $D=5$ where the $y$ dependence is dropped, these results agree with those of [3].

\section{Covariantisation for $D=6$}

Since the procedure should be clear, we just quote the results for $D=6$ in this section. According to the $6+5$ coordinate split the $d=11 \gamma$ matrices are represented by

$$
\begin{array}{ll}
\tilde{\Gamma}^{\alpha}=\gamma^{\alpha} \otimes 1 & \gamma^{\alpha \beta \gamma \delta \varepsilon \zeta}=\varepsilon^{\alpha \beta \gamma \delta \varepsilon \zeta} \Gamma^{*} \\
\tilde{\Gamma}^{a}=\Gamma^{*} \otimes \Gamma^{a} & \Gamma^{a b c d e}=-i \varepsilon^{a b c d e} .
\end{array}
$$

$\gamma^{\alpha}$ and $\Gamma^{a}$ correspond to $\Gamma^{a}$ and $\gamma^{\alpha}$ of the last section with an additional factor of $\mathrm{i}$ due to the metric $\eta^{\alpha \beta}=\operatorname{diag}(+,-, \ldots,-), \eta^{a b}=\operatorname{diag}(-, \ldots,-)$.

In $D=6$ there exist Weyl spinors. The projectors are

$$
\Pi_{ \pm}=\frac{1}{2}\left(1 \pm \Gamma^{*}\right) \otimes 1 .
$$


We want to extend the internal $\mathrm{SO}(5)$ Lorentz group (table 1) so we assign the chiral spinor components (which are still (pseudo)-Majorana spinors) to representations of $\mathrm{SO}(5) \times \mathrm{SO}(5)$ :

$$
\begin{array}{ll}
\varepsilon_{A} \equiv \Pi_{+} \varepsilon^{\prime} & \Psi_{\mu A} \equiv \Pi_{+} \Psi_{\mu}^{\prime} \\
\varepsilon_{\bar{A}} \equiv \Pi_{-} \varepsilon^{\prime} & \Psi_{\mu \bar{A}} \equiv \Pi_{-} \Psi_{\mu}^{\prime} .
\end{array}
$$

$A$ and $\bar{A}$ denote the spinor indices of the first and the second $\mathrm{SO}(5)$ in $\mathrm{SO}(5) \times \mathrm{SO}(5)$, respectively; $a$ and $\bar{a}$ are the corresponding vector indices. The original $\mathrm{SO}(5)$ is, of course, embedded as the diagonal subgroup (just as for $D=3$ [5]).

We redefine the spin- $-\frac{1}{2}$ fields

$$
\begin{aligned}
& \chi_{a \bar{A}} \equiv \frac{1}{2} \Pi_{+} \Gamma^{b} \Gamma_{a} \Psi_{b}^{\prime} \\
& \chi_{\bar{a} A} \equiv \frac{1}{2} \Pi_{-} \Gamma^{b} \Gamma_{a} \Psi_{b}^{\prime}
\end{aligned}
$$

and introduce the '16-bein'

$$
e_{A}^{m}{ }^{\bar{B}} \equiv \mathrm{i} \Delta^{-1 / 4} e_{a}^{m} \Gamma_{A B}^{a} .
$$

This yields $\mathrm{SO}(5) \times \mathrm{SO}(5)$ covariant expressions for the transformation laws (2.5) and (2.7):

$$
\begin{aligned}
& \delta e_{\mu}{ }^{\alpha}=-\frac{1}{2} \mathbf{i}\left(\bar{\varepsilon}^{A} \gamma^{\alpha} \Psi_{\mu A}+\bar{\varepsilon}^{\bar{A}} \gamma^{\alpha} \Psi_{\mu \bar{A}}\right) \\
& \delta B_{\mu}{ }^{m}=-\frac{1}{2} e^{m}{ }_{A}{ }^{\bar{B}}\left[\bar{\varepsilon}^{A} \Gamma^{*} \Psi_{\mu \bar{B}}+\frac{1}{2}\left(\bar{\varepsilon} \gamma_{\mu} \Gamma^{b}\right)^{A} \chi_{b \bar{B}}\right]+(A \leftrightarrow \bar{A}, a \leftrightarrow \bar{a}) .
\end{aligned}
$$

Discarding a local $\mathrm{SO}(5) \times \mathrm{SO}(5)$ rotation we obtain for the supersymmetry transformation of $e^{m}{ }_{A}^{\bar{B}}$ the covariant result

$$
\delta e^{m}{ }_{A}^{\bar{B}}=-\frac{1}{4} \mathrm{i}\left(\bar{\varepsilon}^{C} \Gamma_{a C}{ }^{D} \chi_{\bar{b} D}-\bar{\varepsilon}^{\bar{C}} \Gamma_{\bar{b} \bar{C}}{ }^{\bar{D}} \chi_{a \bar{D}}\right)\left(\Gamma^{a} e^{m} \Gamma^{\bar{b}}\right)_{A}{ }^{\bar{B}} .
$$

One can interpret $(4.8)$ as a (local) $\mathrm{E}_{5(+5)}=\mathrm{SO}(5,5)$ rotation in the following way. The spinor representation of $\mathrm{SO}(5,5)$ has 32 components. In the Weyl basis it is sufficient to consider the 16 upper components. We use the explicit form

$$
\begin{array}{ll}
\hat{\Gamma}_{A \bar{B}, C \bar{D}}^{a b}=-\Gamma_{A C}^{a b} \delta_{\bar{B} \bar{D}} & \hat{\Gamma}_{A \bar{B}, C \bar{D}}^{\bar{a} \bar{b}}=-\Gamma_{\bar{D} \bar{B}}^{\bar{a} \bar{b}} \delta_{A C} \\
\hat{\Gamma}_{A \bar{B}, C \bar{D}}^{a \bar{b}}=\Gamma_{A C}^{a} \Gamma_{\bar{D} \bar{B} .}^{\bar{b}} . &
\end{array}
$$

Acting on $e^{m}{ }_{A}^{\bar{B}}$ the generators $-\hat{\Gamma}_{I J}^{a b}$ and $\hat{\Gamma}_{I J}^{a \bar{b}}(I=A \bar{B}, \ldots)$ give rise to the proper $\mathrm{SO}(5) \times \mathrm{SO}(5)$ transformation $\dagger$. The introduction of $\mathrm{E}_{5(+5)}$ valued fields $(\mathscr{B}, \mathscr{A})$ leads to $\mathrm{SO}(5) \times \mathrm{SO}(5)$ covariant expressions for the fermionic transformation laws. With the abbreviation

$$
F_{\alpha a b}=\varepsilon_{a b c d e} F_{\alpha}^{c d e} \quad F_{a}=\varepsilon_{a b c d e} F^{b c d e}
$$

one finds

$$
\begin{aligned}
& \mathscr{B}_{\mu a b}=-\frac{1}{2} \omega_{\mu a b}+(\sqrt{2} / 24) F_{\mu a b} \\
& \mathscr{B}_{\mu \bar{a} \bar{b}}=-\frac{1}{2} \omega_{\mu a b}-(\sqrt{2} / 24) F_{\mu a b} \\
& \mathscr{A}_{\mu a \bar{b}}=\frac{1}{2} \Omega_{\mu(a b)}-\frac{1}{4} \eta_{a b} \Omega_{\mu c}{ }^{c}+(\sqrt{2} / 24) F_{\mu a b}=-\mathscr{A}_{\mu \bar{b} a} \\
& \mathscr{B}_{m a b}=\frac{1}{2} e_{[a}{ }^{n} \partial_{m} e_{b] n}-(\sqrt{2} / 192) e_{m[a} F_{b]}
\end{aligned}
$$

$\uparrow$ This representation is in fact sixteen dimensional, because we can impose the $\operatorname{SO}(5,5)$ Majorana condition $e_{i}^{m}=\hat{C}_{U}\left(e_{J}^{m}\right)^{*}, \hat{C}_{A \bar{B}, C \bar{D}}=C_{S A C} C_{S}^{-1} \bar{D}_{\bar{B}} \cdot e^{\prime \prime \prime}{ }_{\bar{B}}{ }^{A} \equiv\left(e^{\prime \prime \prime}{ }_{A}{ }^{\bar{B}}\right)^{*}$ transforms in the complex conjugate $\mathrm{SO}(5,5)$ representation. In the gauge $(4.5): e^{m{ }_{A}}{ }^{B}=e^{m i}{ }_{A}$. 


$$
\begin{aligned}
& \mathscr{B}_{m \bar{a} \bar{b}}=\frac{1}{2} e_{[a}{ }^{n} \partial_{m} e_{b] n}+(\sqrt{2} / 192) e_{m[a} F_{b]} \\
& \mathscr{A}_{m a \bar{b}}=\frac{1}{2} e_{(a}{ }^{n} \partial_{m} e_{b) n}-\frac{1}{4} \eta_{a b}\left(\Delta^{-1} \partial_{m} \Delta\right)-(\sqrt{2} / 192) e_{m[a} F_{b]} \\
& \mathscr{B}_{\mu l J}=-\mathscr{B}_{\mu a b} \hat{\Gamma}_{l J}^{a b}+\mathscr{B}_{\mu \bar{a} \bar{b}} \hat{\Gamma}_{l J}^{a \bar{b}} \quad \mathscr{A}_{\mu I J}=\mathscr{A}_{\mu a \bar{\Gamma}} \hat{\Gamma}_{l J}^{a \bar{b}} \quad\left(\mathscr{B}_{m}, \mathscr{A}_{m} \text { analogous }\right) .
\end{aligned}
$$

The 'generalised vielbein postulate' is

$$
\begin{aligned}
& \mathscr{D}_{\mu} e_{I}^{m}+\frac{1}{4} \partial_{n} B_{\mu}{ }^{n} e_{I}^{m}+\partial_{n} B_{\mu}{ }^{m} e_{I}^{n}+\left(\frac{1}{2} \mathscr{B}_{\mu I J}+\mathscr{A}_{\mu I J}\right) e_{J}^{m}=0 \\
& \partial_{m} e_{I}^{n}+\left(\frac{1}{2} \mathscr{B}_{m I J}+\mathscr{A}_{m I J}\right) e_{J}^{n}=0 .
\end{aligned}
$$

Furthermore, we obtain

$$
\begin{gathered}
\mathscr{G}_{\alpha \beta A} \bar{B}=-\frac{1}{4} \Delta^{1 / 4} \Omega_{\alpha \beta a} \Gamma^{a} \Gamma^{*}+(\sqrt{2} / 8) \mathrm{i} \Delta^{-1 / 4} F_{\alpha \beta a b} \Gamma^{a b} \\
+(\sqrt{2} / 96) \mathrm{i} \Delta^{-1 / 4} \varepsilon_{\alpha \beta \gamma \delta \xi \zeta} F^{\gamma \delta \varepsilon \zeta} \Gamma^{*} .
\end{gathered}
$$

In addition, there appears a three-index field strength

$$
\begin{aligned}
& F_{\alpha \beta \gamma a}^{+}=\frac{1}{2}\left(F_{\alpha \beta \gamma a}+\frac{1}{6} \varepsilon_{\alpha \beta \gamma \delta \varepsilon \zeta} F_{a}^{\delta \varepsilon \zeta}\right) \\
& F_{\alpha \beta \gamma \bar{a}}^{-}=\frac{1}{2}\left(F_{\alpha \beta \gamma a}-\frac{1}{6} \varepsilon_{\alpha \beta \gamma \delta \varepsilon \zeta} F_{a}^{\delta \varepsilon \zeta}\right) .
\end{aligned}
$$

Using $\mathrm{SO}(1,5) \times[\mathrm{SO}(5) \times \mathrm{SO}(5)]$ covariant derivatives

$$
\begin{aligned}
& D_{\mu} \varepsilon_{A}=\left[\mathscr{D}_{\mu}-\frac{1}{4} \hat{\omega}_{\mu \alpha \beta} \gamma^{\alpha \beta}\right] \varepsilon_{A}+\frac{1}{2} \mathscr{B}_{\mu a b}\left(\Gamma^{a b} \varepsilon\right)_{A} \\
& D_{m} \varepsilon_{A}=\left[\partial_{m}+\frac{1}{4}\left(e_{\alpha}{ }^{\mu} \partial_{m} e_{\mu \beta}\right) \gamma^{\alpha \beta}\right] \varepsilon_{A}+\frac{1}{2} \mathscr{B}_{m a b}\left(\Gamma^{a b} \varepsilon\right)_{A}
\end{aligned}
$$

(and analogous ones for $A \leftrightarrow \bar{A}, a \leftrightarrow \bar{a}$ ) one arrives at manifestly covariant transformation rules for the fermionic fields:

$$
\begin{aligned}
\delta \Psi_{\mu A}=\left[D_{\mu}(\right. & \left.\left.\hat{\omega}, \mathscr{B}_{\mu}\right)-\frac{1}{8}\left(\partial_{m} B_{\mu}{ }^{m}\right)\right] \varepsilon_{A}+\left(\frac{1}{8} \gamma_{\mu} \gamma^{\alpha \beta}-e_{\mu}{ }^{\alpha} \gamma^{\beta}\right) \mathscr{G}_{\alpha \beta A}{ }^{\bar{B}} \varepsilon_{\bar{B}} \\
& -\frac{1}{4} e^{m}{ }_{A}^{\bar{B}} \Gamma^{*}\left[D_{m}\left(\gamma_{\mu} \varepsilon_{\bar{B}}\right)+\left(\partial_{m} g_{\mu \nu}\right) \gamma^{\nu} \varepsilon_{\bar{B}}\right] \\
& +\frac{1}{8} \gamma_{\mu} A_{m a \bar{b}}\left(\Gamma^{a} e^{m} \Gamma^{\bar{b}}\right)_{A}{ }^{\bar{B}} \varepsilon_{\bar{B}}+(\sqrt{2} / 48) \mathrm{i} \gamma^{\alpha \beta \gamma} \gamma_{\mu} F_{\alpha \beta \gamma d}^{+}\left(\Gamma^{d} \Gamma^{*} \varepsilon\right)_{A} \\
\delta_{\chi \bar{a} A}=\frac{1}{2} e^{m}{ }_{A}{ }^{\bar{B}}( & \left.\Gamma_{\bar{a}} D_{m} \varepsilon\right)_{\bar{B}}+\frac{1}{4} \gamma^{\alpha \beta} \mathscr{G}_{\alpha \beta \bar{B}}{ }_{\bar{B}}\left(\Gamma_{\bar{a}} \Gamma^{*} \varepsilon\right)_{\bar{B}}-A_{m b \bar{a}}\left(\Gamma^{b} e^{m}\right)_{A}{ }^{\bar{B}} \Gamma^{*} \varepsilon_{\bar{B}} \\
& +\frac{1}{4} A_{m b \bar{c}}\left(\Gamma^{b} e^{m} \Gamma^{\bar{c}}\right)_{A}{ }_{\bar{B}}\left(\Gamma_{\bar{a}} \Gamma^{*} \varepsilon\right)_{\bar{B}}-\gamma^{\mu} A_{\mu b \bar{a}}\left(\Gamma^{b} \varepsilon\right)_{A} \\
& +(\sqrt{2} / 24) \mathrm{i} \gamma^{\alpha \beta \gamma} F_{\alpha \beta \gamma \bar{a}}^{-} \varepsilon_{A}
\end{aligned}
$$

$\left(A \leftrightarrow \bar{A}, a \leftrightarrow \bar{a}\right.$ for $\delta \Psi_{\mu \bar{A}}$ and $\left.\delta \chi_{a \bar{A}}\right)$.

\section{Concluding remarks}

The $\mathrm{H}_{11-D}$ (and $\mathrm{E}_{11-D}$ ) structure of $d=11$ supergravity has now been established for $D=3,4,5,6$. After a coordinate split the various components of the $d=11$ graviton and the three-index 'photon' are combined into irreducible representations of the group $\mathrm{H}_{11-D}$. The key ingredient is the generalised vielbein field $[4,5]$

$$
e^{m} \sim \Delta^{-s} e_{a}^{m} \Gamma^{a}
$$

which satisfies a 'generalised vielbein postulate' covariant under global $\mathrm{E}_{11-D}$ rotations. The fermionic redefinitions coincide with those needed to diagonalise the RaritaSchwinger terms after dimensional reduction. One obtains $\mathrm{H}_{11-D}$ covariant expressions for the supersymmetry transformation laws; the covariance of the equations of motion follows by the usual arguments [4]. There is little doubt that the procedure can also be carried out for the remaining values of $D$. For $D=7$ the necessary redefinitions and field assignments can be read off directly from [7]. 
The general coordinate transformations take a rather unusual form in the covariantised versions of $d=11$ supergravity. For the elfbein $E_{M}{ }^{A}$ compensating $\operatorname{SO}(1,10)$ rotations are needed to preserve the gauge $(2.1)$ and a transformation with parameter $\xi^{m}=-B_{\nu}{ }^{m} \xi^{\nu}$ is included [4]. For (5.1) one finds

$\delta_{\mathrm{gc}} e^{m}=\xi^{\mu}\left(\mathscr{D}_{\mu} e^{m}+s \partial_{n} B_{\mu}^{n} e^{m}+\partial_{n} B_{\mu}^{m} e^{n}\right)+\xi^{n} \partial_{n} e^{m}-s \partial_{n} \xi^{n} e^{m}-\partial_{n} \xi^{m} e^{n}$.

Comparing with (3.19) and (4.11) we see that $\left(\mathscr{B}_{\mu}, \mathscr{A}_{\mu}\right)$ generate a $D$-dimensional general coordinate transformation of $e^{m}$. The $(11-D)$-dimensional part of (5.2) can also be expressed as a $\mathrm{E}_{11-D}$ rotation, with parameter $\xi^{m}\left(\frac{1}{2} \mathscr{B}_{m}+\mathscr{A}_{m}\right)(\mathrm{cf}(3.20),(4.12))$ and a corresponding term in which $\xi^{m}\left(e_{a}^{n} \partial_{m} e_{b n}\right)$ is replaced by $e_{a}^{m} \partial_{m} \xi^{n} e_{b n}$.

\section{References}

[1] Cremmer E, Julia B and Scherk J 1978 Phys. Lett. 76B 409

[2] Cremmer E and Julia B 1978 Phys, Lett. 80B 48; 1979 Nucl. Phys. B 159141

[3] Cremmer E 1980 Superspace and Supergravity ed S W Hawking and M Roček (Cambridge: Cambridge University Press) p 267; 1980 Unification of the Fundamental Particle Interactions ed S Ferrara, J Ellis and $\mathbf{P}$ van Nieuwenhuizen (New York: Plenum) p 137

[4] de Wit B and Nicolai H 1985 Phys. Lett. 155B 47; 1986 Nucl. Phys. B 274363

[5] Nicolai H 1987 Phys. Lett. 187B 316

[6] Nicolai H 1987 Phys. Lett. 194B 402

Nicolai $H$ and Warner N P 1988 Preprint CERN TH-5154

[7] Sezgin E and Salam A 1982 Phys. Lett. 118B 359 